\title{
Effect of Allopurinol in Chronic Nonbacterial Prostatitis: A Double Blind Randomized Clinical Trial
}

\author{
Amir M. Ziaee, Hamed Akhavizadegan, Mojgan Karbakhsh \\ Labbafinejad Hospital, Urology Nephrology Research Center, Shahid Beheshti University of \\ Medical Sciences, Tehran, Iran, and Tehran University of Medical Sciences, Tehran, Iran
}

\begin{abstract}
Introduction: The exact mechanism of chronic nonbacterial prostatitis has not been yet elucidated and the outcome with the current management is dismal. In this trial, we studied the effect of allopurinol in the treatment of this disease.

Materials and Methods: In this randomized double blind controlled trial, a calculated sample size of 56 were grouped into "intervention group" who received allopurinol (100 mg tds for 3 months) with ofloxacin (200 mg tds) for 3 weeks ( $=29)$ and "control group" who received placebo tablets with ofloxacin $(n=27)$. Patients' scores based on the National Institute of Health Chronic Prostatitis Symptom Score were recorded before therapy and then every month during the study. A fourglass study was performed before intervention and after 3 months.

Results: The 2 groups were similar regarding outcome variables. In the first month of study, a significant but similar improvement in symptom scores was observed in both groups. Microscopic examination of prostate massage and postmassage samples were also similar in both groups. No side effects due to allopurinol were observed in patients.

Conclusion: We did not find any advantage for allopurinol in the management of chronic prostatitis versus placebo in patients receiving routine antibacterial treatment.
\end{abstract}

Key words: allopurinol; chronic nonbacterial prostatitis; urine reflux

Int Braz. J Urol. 2006; 32: 181-6

\section{INTRODUCTION}

Chronic nonbacterial prostatitis / chronic pelvic pain syndrome (CP/CPPS) is a common reason for urologic visits (1). Despite significant negative impact on patient quality of life (2), the management of the disease has been dismal (3). Because of the heterogeneous nature of this disease, many types of single agents (4) and multimodal therapies (5) have been tried but not proved to be effective. Persson and colleagues hypothesized the role of urate reflux from urine to the prostate in the pathophysiology of the disease for the first time (6) and recommended allopurinol for its treatment in a randomized clinical trial (7). This therapy has not been widely accepted by other urologists because of low response rate reported by others (8). Now in various papers, allopurinol has appeared in the list of potential treatment modalities of chronic prostatitis (9-11). Nevertheless, according to a Cochrane review, provided data are not convincing that allopurinol resulted in the relief of symptoms (12). No other studies have assessed this therapeutic effect. In this study we evaluated the improving effect of allopurinol on clinical signs and symptoms of nonbacterial prostatitis. 


\section{MATERIALS AND METHODS}

This was a double blind randomized controlled trial. To calculate the sample size, we assumed an alpha error of 0.05 , a beta error of 0.2 and the mean scores provided by Persson et al. study (7), the only article similar to ours. In that trial, the mean symptom score between days $45-135$ was -1.08 $(\mathrm{SD}=1.29)$ for the $25 \mathrm{men}$ in the allopurinol group, compared to $-0.21(\mathrm{SD}=0.97)$ for the 14 men in the control group. When the formula of sample size estimation for comparison of 2 means was applied, it was established that the sample size had to be 27 patients per group. Thus, we randomized 56 cases diagnosed with CP/CPPS into 2 groups: intervention $(\mathrm{n}=29)$ and control $(\mathrm{n}=27)$. The patients were recruited from September 2002 to September 2004. All patients were followed to the end of the study (no loss to follow-up). According to the prevailing evidence $(3,13-15)$, the following components were used as the inclusion criteria in this study.

Inclusion criteria - Pain in penis, perineal region, supra pubic, testis and/or pelvis after ejaculation. Voiding symptoms such as dysuria, frequency and sense of incomplete urination. Minimum duration of these symptoms for inclusion in the study was 1 year and minimum total symptom score 14 (moderate severity of symptom). We included only those 20 to 40 years old in order to minimize the effect of BPH on symptom score. A normal abdominal palpation was necessary for inclusion. A classical 4 glass study was performed for each patient which must have been typical for CP/CPPS for being included ( 4 negative cultures and inactive at least for the first 2 specimens) (1).

Exclusion criteria - No past medical history for documented urinary tract infection (positive urine culture, symptoms suggesting acute bacterial prostatitis, upper urinary tract infection and urinary tract tuberculosis), sexually transmitted disease (urethral discharge, genital ulcer and epididymoorchitis), urethral stricture (pelvic fracture, urethral bleeding, urethral instrumentation other than diagnostic cystoscopy and urethral catheterization), neurological disease (vertebral column disease, trauma or surgery, disease affecting nervous system such as multiple sclerosis, cerebrovascular accident), drugs which mimic these symptoms (for example anticholinergics and psychotropics), urinary system disease (tumors, stones and interstitial cystitis diagnosed by cystoscopy or biopsy) and genitourinary system surgery (bladder, kidney, ureter, vasectomy, hernia, varicocelectomy, etc.).

No evidence of neurological disease (gait disturbance, abnormal perineal sensation or anal sphincter tone - a mildly spastic sphincter was considered normal, and spina bifida), genital disease (ulcer, discharge or scar), prostate nodules.

Regarding paraclinics and imaging, normal urine analysis and culture were mandatory. Cases with hematuria or pyuria were excluded from the study. Normal ultrasonography of urinary tract was another essential para-clinical index (no stones, diverticula, masses, abnormally thick bladder wall or post-voiding residue above 50 milliliters).

All the included patients were offered information regarding the explorative nature of the study and consented by written agreement. They were interviewed before any medical interventions and then monthly for 3 months using the National Institute of Health (NIH) prostatitis symptom index (13) translated into Farsi. Translation and back translation was made by 2 of the authors; one of whom did the translation and the other who did not know the original English text did the back translation. The final translation was fixed by consensus of all authors and was ready to the patients to facilitate communication of symptoms and improve response rate.

The intervention group received allopurinol $100 \mathrm{mg}$ three-times-daily (tds) for 3 months in addition to ofloxacin for the 3 first weeks and the control group received placebo tablets (manufactured exactly similar to the color and shape of allopurinol tablets for the purpose of this trial) and ofloxacin. The rationale for ofloxacin usage was being the recommended drug for chronic nonbacterial prostatitis management, covering culture-negative germs like clamydia (3) and the dosage (200 $\mathrm{mg}$ tds instead of $300 \mathrm{mg}$ bid) was chosen to improve compliance (as allopurinol/placebo were also prescribed tds) (16).

Pain score, urinary symptom score, quality of life score and total symptom score (the primary 
major outcome) were recorded four times for each patient: once before treatment and three times afterwards in one-month intervals. In the case of patients' participation, the four-glass test was repeated at the end of the trial. The patients were also requested a 24-hour urine collection for creatinine and uric acid before and after the treatment. Age, duration of current disease, history of alpha-blocker intake and its response, four glass results and symptom index were recorded for patients.

Scores numerated from baseline through 3 (e.g. total score baseline, total score 1) refer to scores before intervention (0) and at the corresponding months of drug administration.

In each visit, patients were asked about any side effects (jaundice, pruritus, rash, and edema).

General Linear Model (repeated measures) in SPSS 11.5 was used for statistical analysis. $\mathrm{P}=$ 0.05 was considered as the level of statistical significance.

\section{RESULTS}

Mean and standard deviation of age was 33.39 \pm 6.2 . Comparison of underlying variables between 2 groups before intervention showed no statistical differences (Table-1).
No significant differences between the 2 treatment groups on the study scores were observed ("no between-group effect") (Table-2). Nevertheless, significant differences were detected at the end of the first month "within" each group $\left(\mathrm{P}_{\text {pain score }}=0.001, \mathrm{P}_{\text {urinary }}\right.$ score $=0.05, \mathrm{P}_{\text {quality of life score }} \leq 0.001$ and $\mathrm{P}_{\text {total score }} \leq 0.001$ ). Therefore, the symptom scores decreased nearly 30 percent in the first month of study in both groups with no significant changes following (Figure-1).

The white blood cells content in 4-glass test and 24-hour urine collection for uric acid showed no significant differences, neither within nor between the 2 treatment groups. No side effects of allopurinol were detected in intervention group.

\section{COMMENTS}

Only one small trial of allopurinol for treatment of chronic prostatitis has shown improvements in patient-reported symptoms, investigator-graded prostate pain and biochemical parameters to date (7); but no other evidence exists to support it (9). In that very research (7), 54 patients (with 39 patients completing the study) were randomized into 2 groups (placebo and allopurinol) with significant improvement in the intervention group.

Table 1 - Comparison of underlying variables between 2 groups (intervention and control) before treatment.

\begin{tabular}{lccc}
\hline Variable/Group & Intervention Group & Control Group & p Value \\
\hline Age (mean \pm SD) & $33.28 \pm 6.4$ & $33.52 \pm 6.15$ & 0.89 \\
History of alpha-blocker usage (\%) & 55.2 & 63 & 0.55 \\
Good response to alpha-blocker (\%) & 00 & 01.8 & 0.31 \\
$>10$ WBC/HPF in EPS (\%) & 35.7 & 26.9 & 0.49 \\
$>$ 10 WBC/HPF in VB3 (\%) & 21.4 & 26.9 & 0.64 \\
Pain score (mean \pm SD) & $11.48 \pm 2.87$ & $10.37 \pm 4.61$ & 0.28 \\
Voiding symptom score (mean \pm SD) & $06.69 \pm 3.2$ & $05.70 \pm 3.9$ & 0.3 \\
Quality of life score (mean \pm SD) & $08.10 \pm 2.24$ & $8.370 \pm 0.2$ & 0.64 \\
Pain plus voiding score (mean \pm SD) & $18.17 \pm 4.38$ & $16.70 \pm 5.45$ & 0.27 \\
Total score (mean \pm SD) & $26.28 \pm 5.5$ & $25.07 \pm 6.53$ & 0.46 \\
\hline
\end{tabular}

$W B C=$ white blood cells; HPF = high power field. 


\section{Allopurinol in Chronic Nonbacterial Prostatitis}

Table 2 - Mean and standard deviation of symptom scores in the 2 study groups.

\begin{tabular}{|c|c|c|c|c|c|c|}
\hline \multirow[t]{2}{*}{ Scores $($ mean \pm SD $)$} & \multirow[t]{2}{*}{ Group } & \multicolumn{4}{|c|}{ Time Interval } & \multirow[t]{2}{*}{ p Value } \\
\hline & & Baseline & Month 1 & Month 2 & Month 3 & \\
\hline \multirow[t]{2}{*}{ Pain symptom score } & allopurinol & $12.04 \pm 2.66$ & $8.66 \pm 4.66$ & $6.96 \pm 4.38$ & $7.62 \pm 4.37$ & \multirow[t]{2}{*}{0.65} \\
\hline & placebo & $9.65 \pm 4.57$ & $8.17 \pm 4.46$ & $7.82 \pm 5.04$ & $7.73 \pm 4.25$ & \\
\hline \multirow[t]{2}{*}{ Urinary symptom score } & allopurinol & $6.96 \pm 3.34$ & $4.46 \pm 3.61$ & $5.31 \pm 7.80$ & $4.16 \pm 2.82$ & \multirow[t]{2}{*}{0.142} \\
\hline & placebo & $5.61 \pm 3.83$ & $3.56 \pm 3.36$ & $3.52 \pm 3.34$ & $3.39 \pm 3.07$ & \\
\hline \multirow[t]{2}{*}{ Quality of life symptom score } & allopurinol & $8.33 \pm 2.16$ & $5.41 \pm 2.65$ & $5.41 \pm 2.6$ & $5.21 \pm 2.84$ & \multirow[t]{2}{*}{0.42} \\
\hline & placebo & $8.43 \pm 1.97$ & $6 \pm 2.28$ & $6 \pm 2.95$ & $65.87 \pm 2.75$ & \\
\hline \multirow[t]{2}{*}{ Total symptom score } & allopurinol & $27.33 \pm 5.21$ & $18.54 \pm 9.09$ & $16.29 \pm 7.50$ & $17 \pm 7.76$ & \multirow[t]{2}{*}{0.85} \\
\hline & placebo & $24.43 \pm 6.46$ & $17.95 \pm 7.6$ & $18.13 \pm 9.61$ & $17.21 \pm 8.5$ & \\
\hline
\end{tabular}

*p value of between group effects.

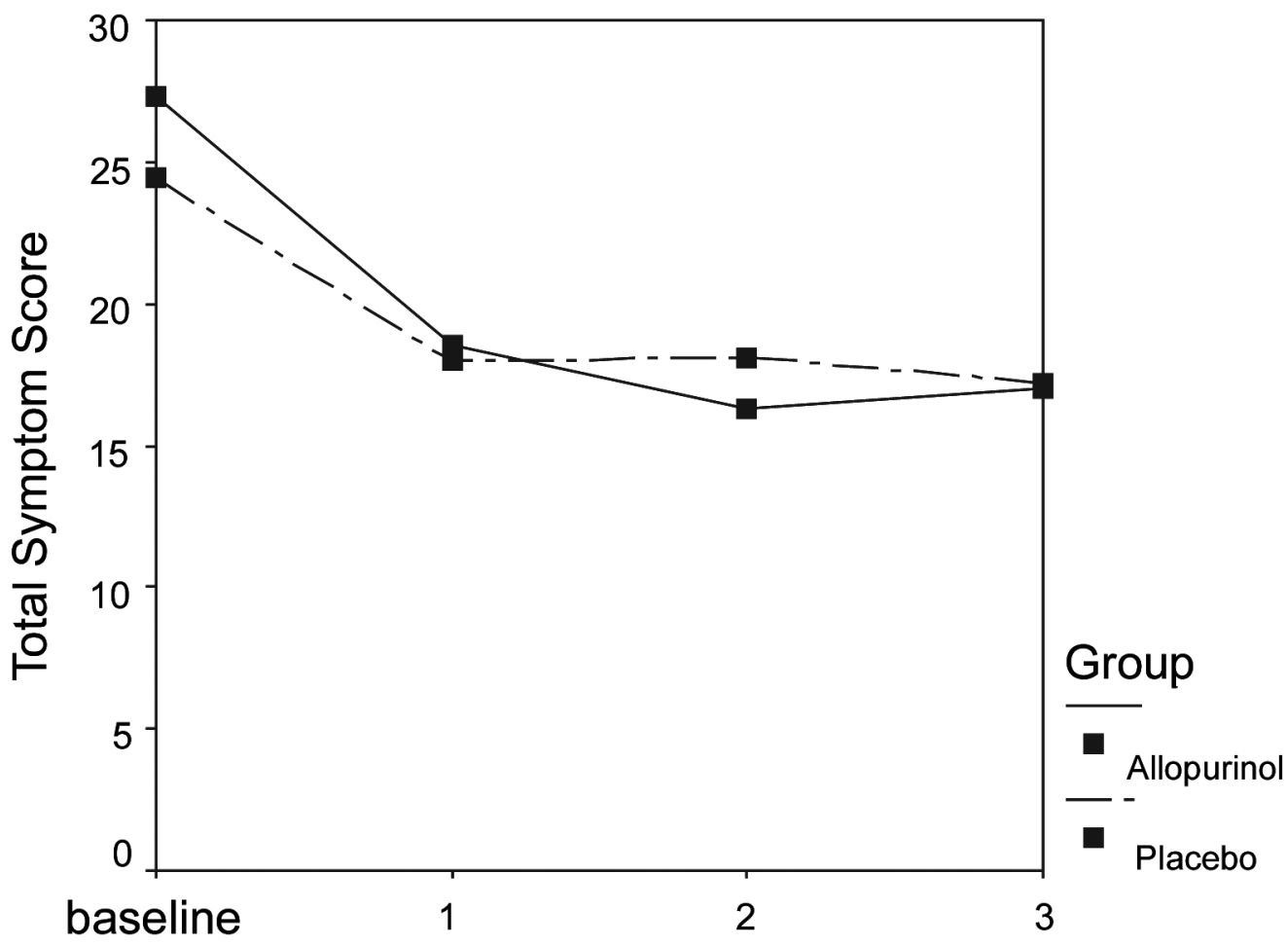

Months of the Study

Figure 1 - Mean of total symptom score at different months of the study in the 2 groups. 
Our study was designed in line with the CP/ CPPS clinical trial reported by the National Institutes of Health Chronic Prostatitis Collaborative Research Network (13). The NIH/ symptom score (17), which is a valid questionnaire (18-21) for CP/CPPS, has been used for scoring the prostatitis symptoms. Persson and colleagues (7), using their own questionnaire, observed the peak ameliorative effect of allopurinol after three months. The three-month period for followup was decided on this basis in our trial.

In this study, we did not find any differences between "allopurinol and ofloxacin" and "placebo and ofloxacin" in treating CP/CPPS. In the first month of follow up, symptoms improved significantly in both groups. Nevertheless, no further improvement was observed in the intervention group in comparison with the control groups. The improvement of all symptom indices in the first month might be attributed to initial placebo effect or elimination of culture-negative germs, with the latter hypothesis being rather farfetched: in that case, we have to consider "chronic bacterial prostatitis" as the main etiology of our patients' symptoms, an otherwise uncommon condition (22).

Persson's paper was the only study reporting the effect of allopurinol on CP/CPPS. In his study, there were some methodological limitations. Some patients were not in the active phase of disease, some had positive cultures, some were lost in follow up, white blood cells in 4 glass test was not measured directly and some of the symptom scores and $\mathrm{P}$ value were not reported (8). Because of these shortcomings and lack of any other supporting studies, it has been difficult to verify the effect of allopurinol in chronic nonbacterial prostatitis (12). In this study we tried to overcome these methodological shortcomings. Nevertheless, we did not find any preference for allopurinol to placebo in CP/CPPS management.

Our study has some limitations: first, the possibility of selection bias: although according to the related literature, urine analysis and culture, ultrasonography and four-glass test are considered enough to confirm the diagnosis of chronic nonbacterial prostatitis $(3,14,15)$, it is still probable that some patients with other diseases - mimicking chronic nonbacterial prostatitis symptoms - have been included in our study (16). Second, the low power of the study due to low number of patients recruited, according to the calculated sample size. Nevertheless, the probability that a significant difference really exists is very low considering the very similar results in the two groups. Third, antibiotic usage in both groups, which is generally recommended in cases of chronic prostatitis, may make it difficult to interpret the first-month improvement in patients' symptoms.

\section{CONCLUSION}

Our study showed that allopurinol does not have any ameliorative effect on chronic nonbacterial prostatitis regarding clinical symptoms or improvement of quality of life in comparison with placebo. This disease or syndrome has a collection of symptoms with unknown origins. These symptoms may have diverse etiologies and thus a small subgroup may benefit from allopurinol but we do not recommend the routine use of allopurinol for treatment of CP/CPPS.

\section{CONFLICT OF INTEREST}

None declared.

\section{REFERENCES}

1. Batstone GR, Doble A: Chronic prostatitis. Curr Opin Urol. 2003; 13: 23-9. Review. Erratum in: Curr Opin Urol. 2003; 13: 177. Batstone D [corrected to Batstone G Richard D].

2. Turner JA, Ciol MA, Von Korff M, Berger R: Prognosis of patients with new prostatitis/pelvic pain syndrome episodes. J Urol. 2004; 172: 538-41.

3. Nickel JC, Downey J, Ardern D, Clark J, Nickel K: Failure of a monotherapy strategy for difficult chronic prostatitis/chronic pelvic pain syndrome. J Urol. 2004; 172: 551-4.

4. Krieger JN: The problem with prostatitis. What do we know? What do we need to know? J Urol. 2004; 172: 432-3.

5. Shoskes DA, Hakim L, Ghoniem G, Jackson CL: Longterm results of multimodal therapy for chronic 
prostatitis/chronic pelvic pain syndrome. J Urol. 2003; 169: 1406-10.

6. Persson BE, Ronquist G: Evidence for a mechanistic association between nonbacterial prostatitis and levels of urate and creatinine in expressed prostatic secretion. J Urol. 1996; 155: 958-60.

7. Persson BE, Ronquist G, Ekblom M: Ameliorative effect of allopurinol on nonbacterial prostatitis: a parallel double-blind controlled study. J Urol. 1996; 155: 961-4.

8. Nickel JC, Siemens DR, Lundie MJ: Re: Ameliorative effect of allopurinol on nonbacterial prostatitis: a parallel double-blind controlled study. J Urol. 1997; 157: 628-9.

9. Stevermer JJ, Easley SK: Treatment of prostatitis. Am Fam Physician. 2000; 61: 3015-22, 3025-6. Erratum in: Am Fam Physician 2001; 63: 2129.

10. Naber KG, Weidner W: Chronic prostatitis-an infectious disease? J Antimicrob Chemother. 2000; 46: 157-61.

11. Doble A: An evidence-based approach to the treatment of prostatitis: is it possible? Curr Urol Rep. 2000; 1: 142-7.

12. McNaughton $\mathrm{CO}$, Wilt T: Allopurinol for chronic prostatitis. Cochrane Database Syst Rev. 2002; 4: CD001041.

13. Propert KJ, Alexander RB, Nickel JC, Kusek JW, Litwin MS, Landis JR, et al.: Design of a multicenter randomized clinical trial for chronic prostatitis/chronic pelvic pain syndrome. Urology. 2002; 59: 870-6.

14. Kaplan SA, Volpe MA, Te AE. A Prospective, 1-Year Trial Using Saw Palmetto Versus Finasteride in the Treatment of Category III Prostatitis/Chronic Pelvic Pain Syndrome. J Urol. 2004; 171: 284-8.

15. Nickel JC, Downey J, Pontari MA, Shoskes DA, Zeitlin SI: A randomized placebo-controlled multicentre study to evaluate the safety and efficacy of finasteride for male chronic pelvic pain syndrome (category IIIA chronic nonbacterial prostatitis). BJU Int. 2004; 93: 991-5.

16. McNaughton Collins M, MacDonald R, Wilt TJ: Diagnosis and treatment of chronic abacterial prostatitis: a systematic review. Ann Intern Med. 2000; 133: 367-81.

17. Litwin MS, McNaughton-Collins M, Fowler FJ Jr, Nickel JC, Calhoun EA, Pontari MA, et al.: The National Institutes of Health chronic prostatitis symptom index: development and validation of a new outcome measure. Chronic Prostatitis Collaborative Research Network. J Urol. 1999; 162: 369-75.

18. Turner JA, Ciol MA, Von Korff M, Berger R: Validity and responsiveness of the national institutes of health chronic prostatitis symptom index. J Urol. 2003; 169: 580-3.

19. Collins MM, O'Leary MP, Calhoun EA, Pontari MA, Adler A, Eremenco S, et al.: The Spanish National Institutes of Health-Chronic Prostatitis Symptom Index: translation and linguistic validation. J Urol. 2001; 166: 1800-3.

20. Leskinen MJ, Mehik A, Sarpola A, Tammela TL, Jarvelin MR: The Finnish version of The National Institutes Of Health Chronic Prostatitis Symptom Index correlates well with the visual pain scale: translation and results of a modified linguistic validation study. BJU Int. 2003; 92: 251-6.

21. Nickel JC: Prostatitis: lessons from the 20th century. BJU Int. 2000; 85: 179-85.

22. Usupbaev AC, Hakimhodjaev ZS, Kim AS. The new diagnostic approaches in assessment of abacterial forms (category III) of the chronic prostatitis. BJU Int September 2002: Vol 90; Suppl 2, pp. 226-7.

\section{Correspondence address:}

Dr. Amir Mohsen Ziaee

Shahid Dr. Labbafinegad Hospital

9th Boostan Street, Pasdaran Avenue, Tehran, Iran

Fax: + 9821 254-9088

E-mail: ziaee@hotmail.com 